\title{
Performance Enhancement of Differential UWB Autocorrelation Receivers Under ISI
}

\author{
Marco Pausini, Student Member, IEEE, Gerard J. M. Janssen, Member, IEEE, and Klaus Witrisal, Member, IEEE
}

\begin{abstract}
The autocorrelation receiver (AcR) is a suboptimum, low-complexity receiver architecture particularly suited to ultra-wideband (UWB) communication systems. As the bit rate increases, interference among pulses due to multipath propagation causes serious impairments of the AcR's performance. To mitigate this effect, we propose an appropriate design of the chip code and of the delay hopping (DH) code. We provide conditions to be satisfied by the DH code in order to reduce the nonlinear intersymbol interference (ISI) and the bias term, which are peculiar nuisance parameters of autocorrelation receivers. By extending the length of the chip code, we show that $N_{p}$ transmitted pulses per symbol can be employed to suppress the average linear ISI of $N_{p}-1$ previous symbols. Simulated results confirm the performance improvement in terms of bit-error rate. However, in previous work it has been shown that the noise power linearly increases with $N_{p}$. Although a large number of pulses per symbol is favorable for ISI mitigation, we show that the transmission of a single pulse minimizes the probability of error, for bit rates lower than an upper bound depending on the channel root mean-square delay spread and on the noise power.
\end{abstract}

Index Terms-Autocorrelation receiver, chip and delay hopping (DH) code, intersymbol interference (ISI), number of pulses per symbol, ultra-wideband (UWB).

\section{INTRODUCTION}

$\mathbf{U}$ LTRA-WIDEBAND (UWB) impulse radio (IR) systems operate by transmitting a train of subnanosecond pulses, occupying a bandwidth of a few gigahertz, which allows an extremely fine time-resolution capability. Thus, many distinct propagation paths can be resolved, resulting in a reduction of fading, when optimum receivers are employed. However, the implementation complexity of a RAKE receiver with tens or even hundreds of fingers, performing accurate acquisition and channel estimation, seems to be unaffordable. For these reasons, suboptimal, low-complexity autocorrelation receivers (AcR's) proposed 40 years ago [2], [3], have regained popularity.

In UWB AcRs, the received signal consisting of a train of pulses is delayed and correlated with itself, using a set of delaylines plus correlators, and sampled at the bit rate. The delayed

Manuscript received February 28, 2005; revised October 13, 2005. This work was supported in part by the Dutch Ministry of Economic Affairs/Ministry of Education Freeband-Impulse Project Airlink. This paper was presented in part at the IEEE International Conference on Communications, Seoul, Korea, May 2005.

M. Pausini and G. J. M. Janssen are with the Wireless and Mobile Communications Group, Delft University of Technology, 2628 CD Delft, The Netherlands (e-mail: M.Pausini@ewi.tudelft.nl; G.Janssen@ewi.tudelft.nl).

K. Witrisal is with the Signal Processing and Speech Communication Laboratory, Graz University of Technology, 8010 Graz, Austria (e-mail: Witrisal@ tugraz.at).

Digital Object Identifier 10.1109/JSAC.2005.863845 signal is used as a template in the demodulation block without requiring any kind of channel estimation. Thus, one pulse is used to "sound" the channel, and another one is used to convey data. Data can be applied, for instance, by changing the polarity of the "data" pulse with regard to the "reference" pulse, and the overall system is usually referred to as transmitted reference (TR) AcR [4], [5].

However, the transmission of a reference pulse for each data pulse is clearly inefficient. In this paper, we consider the frame differential (FD) AcR [6], where each pulse is differentially modulated with respect to the previous one, and acts as reference for the next one. The total number of pulses per symbol, required for a certain performance quality, is therefore halved compared to the TR scheme. Differential schemes are also proposed in [7]-[9].

Because the AcR obtains the signal template for demodulation from the received signal itself, the noise power is enhanced by an extra noise-to-noise term. In addition, the interference among pulses due to the multipath channel propagation has a more severe impact than in optimum receivers, where the template signal is interference-free. The effects of this extra-interference have been studied in [6], where the observed variable has been modeled by a nonlinear second-order Volterra system [10], as presented in Section II. This model shows the presence of a bias term and distinguishes the ISI in linear and nonlinear data dependent terms.

In Section III, we first show that the nonlinear data interference and the bias term can be mitigated via an appropriate time-positioning of the pulses, expressed analytically by a set of conditions imposed on the delay hopping (DH) code. Then, the length of the chip code is extended and the polarities of the $N_{p}$ pulses transmitted per symbol waveform are exploited to significantly reduce the linear ISI, resulting from $N_{p}-1$ interfering past symbols. Thus, large values of $N_{p}$ on the one hand lead to ISI mitigation, and on the other hand to an increased level of the noise power, as shown in [1], where a linear proportionality between the power of the noise-to-noise term and the transmitted number of pulses per symbol $N_{p}$ has been observed.

In Section IV, we prove that the transmission of one pulse per symbol minimizes the bit-error rate (BER) for values of the bit rate lower than an upper bound, which depends on the channel delay spread and the ratio between linear and no-linear noise power. However, this upper bound is a sufficient but not necessary condition, which means that even if the bit rate exceeds this threshold, a single pulse per symbol can still guarantee the best receiver performance.

In Section V, we show how to further enhance the performance of the one-pulse-per symbol AcR, by designing a 
DH code with properties derived in Section III. Finally, in Section VI, conclusions are drawn.

Notation: We use upper (lower) bold face letters to denote matrices (column vectors), and calligraphic letters $\mathcal{X}$ to denote sets of elements. Superscript ${ }^{T}$ represents transpose. Symbol mod represents the modulo operator, and $\lfloor x\rfloor$ the largest integer $\leq x$. Next, $[\mathbf{X}]_{q, r}$ denotes the matrix entry at row $q$ and column $r$.

\section{SYSTEM MODEL}

\section{A. Frame Differential Modulation}

The transmitted signal waveform for the FD-AcR is

$$
s(t)=\sum_{n=-\infty}^{\infty} \sum_{j=0}^{N_{p}-1} a_{n, j} w^{\prime}\left(t-t_{n, j}\right)
$$

where $w^{\prime}(t)$ is the transmitted pulse, of duration $T_{w}$, and $n, j$ are, respectively, the symbol and frame indexes. The time-instants

$$
t_{n, j}=\left(n N_{p}+j\right) T_{f}+c_{n, j} T_{c}
$$

define the position of the pulses, where $T_{f}$ is the average spacing between two pulses, usually named frame time and $T_{c}$ is the pulse position resolution. Each symbol consists of $N_{p}$ pulses, and thus the symbol duration is equal to $T_{s}=N_{p} T_{f}$. Each pulse is pseudorandomly placed within the time frame via the timehopping (TH) code $c_{n, j} \in\left\{0,1, \ldots N_{c}-1\right\}, N_{c}=\left\lfloor T_{f} / T_{c}\right\rfloor$. The code is repeated every $M$ symbols, and the overall TH code periodicity is therefore $N_{d}=N_{p} \times M$ frames, and the notation used in (2) implicitly assumes $c_{n, j}=c_{n \bmod M, j}$. The pulse amplitude is given by

$$
a_{n, j+1}=a_{n, j} b_{n, j} d_{n}
$$

where $a_{n+1,0} \triangleq a_{n, N_{p}}, d_{n} \in\{-1,+1\}$ is the data symbol and $b_{n, j} \in\{-1,+1\}$ is a chip code of periodicity $L$ symbols, and equivalently $L \times N_{p}$ frames. In Section III, it will be shown that a chip code with $L>1$ can be exploited to reduce ISI. The resulting modulation (3) is then a frame differential scheme, since the data is differentially modulated via consecutive pulses (frames), delayed by the DH code $D_{n, j}=t_{n, j+1}-t_{n, j}$. Similarly, to the notation adopted for TH code elements $c_{n, j}$, in (3) and in the following, the paper holds $b_{n, j}=b_{n \bmod L, j}$, $D_{n, j}=D_{n \bmod M, j}$.

\section{B. Autocorrelation Receiver}

The received signal, after a time-invariant multipath channel $h(t)$ and corrupted by additive white Gaussian noise (AWGN) $n(t)$, is passed through a filter with impulse response $f(t)$ and with bandwidth $W$, to eliminate the out-of-band noise. Thus, we obtain

$$
\hat{r}(t)=\sum_{n=-\infty}^{\infty} \sum_{j=0}^{N_{p}-1} a_{n, j} g\left(t-t_{n, j}\right)+v(t)
$$

where $g(t) \triangleq w^{\prime}(t) * h(t) * f(t)$ and $v(t) \triangleq n(t) * f(t)$. The channel response is modeled as a sum of delta-pulses, $h(t)=$ $\sum_{m=0}^{\infty} \alpha_{m} \delta\left(t-\nu_{m}\right)$, where $\alpha_{m}$ are independent zero-mean random variables. The variance of the ray amplitudes $\alpha_{m}$ decreases exponentially as a function of the time delays $\nu_{m}$.

The FD-AcR consists of $N_{d}$ correlators. At each correlator a delayed replica of the received signal, matched to the lag $D_{n, j}$, is correlated with the signal itself over a time interval $T_{I}$, and the output without noise of each correlator is given by

$$
y_{j}[i]=\int_{t_{i, j}}^{t_{i, j}+T_{I}} r(t) r\left(t+D_{i, j}\right) d t
$$

where $r(t)=\hat{r}(t)-v(t)$ is the noise-free received signal. The outputs $y_{j}[i], j=0, \ldots, N_{p}-1$ are coherently combined to form the decision variable $z[i]=\sum_{j=0}^{N_{p}-1} b_{i, j} y_{j}[i]$.

If we denote with $\eta$ the number of the interfering past symbols, and we assume $T_{I} \leq T_{s}$, then we may collect the polarities of all the pulses falling in the observation window $\left[t_{i, j}, t_{i, j}+T_{I}\right]$ in the vector $\mathbf{a}[i]=\left[a_{i-\eta, 0} \ldots a_{i+1, N_{p}-1}\right]^{T}$ (see Fig. 1). So far, each pulse has been uniquely labeled by using one index for the symbol and one index for the frame. Alternatively, we may adopt only one index $q=0,1, \ldots$, which counts the pulse number starting from a reference pulse. In the following, we choose the pulse of amplitude $a_{i-\eta, 0}$ and time-position $t_{i-\eta, 0}$ as reference. Then, we may use for the amplitude and the time-position of each pulse the following notation:

$$
\begin{aligned}
a_{i-\eta, q} & \triangleq a_{i-\eta+\left\lfloor q / N_{p}\right\rfloor, q \bmod N_{p}} \\
t_{i-\eta, q} & \triangleq t_{i-\eta+\left\lfloor q / N_{p}\right\rfloor, q \bmod N_{p}} .
\end{aligned}
$$

The same notation is applied to the DH code and to the chip code, so that

$$
\begin{aligned}
b_{i-\eta, q} & \triangleq b_{i-\eta+\left\lfloor q / N_{p}\right\rfloor, q \bmod N_{p}}, \\
D_{i-\eta, q} & \triangleq D_{i-\eta+\left\lfloor q / N_{p}\right\rfloor, q \bmod N_{p}}
\end{aligned}
$$

and we recall $b_{n, j}=b_{n \bmod L, j}, D_{n, j}=D_{n \bmod M, j}$. Once a reference timing is fixed, the pulse-timing description in terms of the TH code and in terms of DH code are equivalent, and we can write

$$
t_{i-\eta, q}=t_{i-\eta, 0}+\sum_{q^{\prime}=0}^{q-1} D_{i-\eta, q^{\prime}} .
$$

Regardless of the adopted formalism, we obtain for the correlator output $j$ the quadratic form

$$
y_{j}[i]=\mathbf{a}^{T}[i] \mathbf{Y}_{i, j} \mathbf{a}[i]
$$

where each element of the matrix $\mathbf{Y}_{i, j}$ is given as

$$
\begin{aligned}
& {\left[\mathbf{Y}_{i, j}\right]_{q+1, r+1}=\int_{t_{i, j}}^{t_{i, j}+T_{I}} g\left(t-t_{i-\eta, q}\right) } \\
& \times g\left(t-t_{i-\eta, r}+D_{i, j}\right) d t
\end{aligned}
$$

which represents the autocorrelation of the channel response of pulse $a_{i-\eta, q}$ with the pulse $a_{i-\eta, r}$, the latter time-shifted by the lag $D_{i, j}$, and where $q, r=0, \ldots,(\eta+2) N_{p}-1$. Because of the differential modulation format, we may refer the polarities of all the pulses to the reference $a_{i-\eta, 0}$. If we assume $N_{p}$ to be even, we obtain

$$
a_{i-\eta, q}= \begin{cases}a_{i-\eta, 0} \prod_{l=0}^{q-1} b_{i-\eta, l} & q \bmod 2=0, \\ a_{i-\eta, 0} \prod_{l=0}^{q-1} b_{i-\eta, l} d_{i-\eta+\left\lfloor q / N_{p}\right\rfloor} & q \bmod 2=1 .\end{cases}
$$




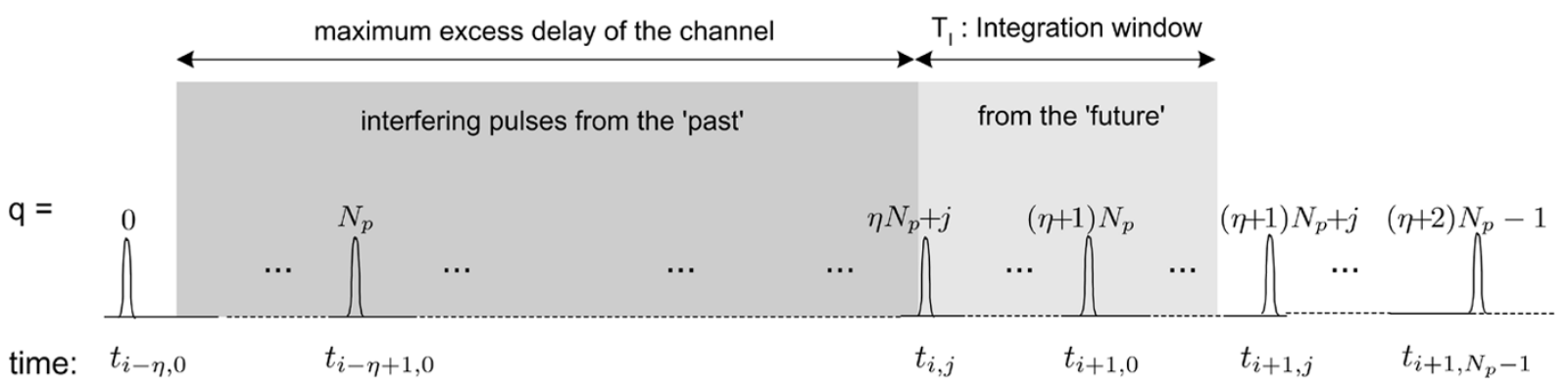

Fig. 1. Transmitted signal and range of interfering pulses at receiver branch $j$.

This equation basically says that the pulse polarity is data independent if $q$ is even, and data dependent, otherwise. It follows that $a_{i-\eta, q} a_{i-\eta, r}$ could be data independent, linearly data dependent, or nonlinearly data dependent. The correlator output $y_{j}[i]$ can therefore be described by a second-order Volterra system, given by $y_{j}[i]=\xi_{i, j}+\mathbf{h}_{i, j}^{T} \mathbf{d}[i]+\mathbf{d}^{T}[i] \mathbf{H}_{i, j} \mathbf{d}[i]$ (see $[6])$, where $\mathbf{d}[i] \triangleq\left[d_{i-\eta} \ldots d_{i+1}\right]$. The observed variable $z[i]$ is finally given by

$$
z[i]=\sum_{j=0}^{N_{p}-1} b_{i, j} y_{j}[i]=\xi_{i}+\mathbf{h}_{i}^{T} \mathbf{d}[i]+\mathbf{d}^{T}[i] \mathbf{H}_{i} \mathbf{d}[i] .
$$

The bias term $\xi_{i}$, the linear ISI coefficients $\mathbf{h}_{i}$ and the nonlinear ISI coefficients $\mathbf{H}_{i}$ are time-dependent with periodicity $M \times$ $L$ and given by a linear combination of disjoint subsets of the matrix $\mathbf{Z}_{i}=\sum_{j=0}^{N_{p}-1} b_{i, j} \mathbf{Y}_{i, j}$ elements. The exact structure of each of these coefficients is presented in [6].

\section{INTERSYMBOL INTERFERENCE (ISI)}

By writing the vector $\mathbf{h}_{i, j}$ of $\eta+2$ elements, as

$$
\mathbf{h}_{i, j}=\left[h_{i, j}(-\eta) \ldots h_{i, j}(0) \quad h_{i, j}(1)\right]^{T}
$$

and recalling that $\mathbf{h}_{i}=\sum_{j=0}^{N_{p}-1} b_{i, j} \mathbf{h}_{i, j}$, we observe that the term $h_{i}(0)=\sum_{j} b_{i, j} h_{i, j}(0)$ represents the useful energy for the detection of the data $d_{i}$, and all the other elements of $\mathbf{h}_{i}$ are the linear-data ISI coefficient. The coefficient $\xi_{i}=\sum_{j} b_{i, j} \xi_{i, j}$ is a bias term which shifts the optimum threshold, and the elements of $\mathbf{H}_{i}=\sum_{j} b_{i, j} \mathbf{H}_{i, j}$ are the nonlinear-data ISI coefficients, all of them peculiar nuisances in the FD-AcR. Because of the random nature of the radio channel, all these quantities can be modeled as random variables.

In [11], we studied the statistical properties till the second order of

$$
I\left(\alpha, T_{I} ; \tau\right) \triangleq \int_{\alpha}^{\alpha+T_{I}} g(t) g(t+\tau) d t, \quad \tau \geq 0
$$

and the analytical results have been validated through a comparison with measured data in [12]. By defining $\tau: \mathcal{A} \rightarrow \mathbb{R}$, $\mathcal{A}=\left\{(q, r) \mid q=0, \ldots(\eta+2) N_{p}-1, r=0, \ldots(\eta+2) N_{p}-1\right\}$, $\mathbb{R}$ the set of the real numbers and $\tau_{q, r} \triangleq t_{i-\eta, r}-t_{i-\eta, q}$, we can write

$$
\begin{aligned}
& {\left[\mathbf{Y}_{i, j}\right]_{q+1, r+1}} \\
& \quad= \begin{cases}I\left(t_{i, j+1}-t_{i-\eta, r}, T_{I} ; \tau_{q, r}-D_{j}\right) & \tau_{q, r} \geq D_{j} \\
I\left(t_{i, j}-t_{i-\eta, q}, T_{I} ; D_{j}-\tau_{q, r}\right) & \tau_{q, r}<D_{j}\end{cases}
\end{aligned}
$$

From this equation, and applying the results obtained in [11], we can derive the expected value and variance of all the parameters of the Volterra system. With regard to the expected values computation, for a NLOS channel the following approximation holds [11]:

$$
E\left\{I\left(\alpha, T_{I} ; \tau\right)\right\} \approx \phi_{w}(\tau) e^{-\gamma \alpha}\left(1-e^{-\gamma T_{I}}\right), \quad \alpha \geq 0
$$

where $\gamma=1 / \tau_{R M S}$, with $\tau_{R M S}$ denoting the RMS delay spread of the channel, and where $\phi_{w}(\tau)=\int_{-\infty}^{\infty} w(t) w(t+\tau) d t$ is the autocorrelation function of $w(t)=w^{\prime}(t) * f(t)$. We define $E_{w} \triangleq \phi_{w}(0)$.

\section{A. Delay Hopping (DH) Code Design}

Applying (16) to(15), we first observe that

$$
E\left\{\left[\mathbf{Y}_{i, j}\right]_{q+1, r+1}\right\}=0 \Leftrightarrow \phi_{w}\left(\left|\tau_{q, r}-D_{i, j}\right|\right)=0 .
$$

Then, we notice that the correlation lag $\tau_{q, r}-D_{i, j}$ at the receiver branch $j$ between the pulses $a_{i-\eta, q}$ and $a_{i-\eta, r}$, assumes only values which are multiple integers of the time-pulse resolution $T_{c}$. Thus, if we choose $T_{c}$ such that $\phi_{w}\left(T_{c}\right)=0$ (e.g., $T_{c}$ greater than the pulse duration $T_{w}$ ), we can rewrite (17) as

$$
E\left\{\left[\mathbf{Y}_{i, j}\right]_{q+1, r+1}\right\}=0 \Leftrightarrow\left|\tau_{q, r}\right| \neq D_{i, j} .
$$

From (10), we can write the time delay between the pulses of index $r$ and $q$ as $\tau_{q, r}=\sum_{l=q}^{r-1} D_{i-\eta, l}$, with $r>q$. By definition, the adjacent pulse-pairs $a_{i, j}, a_{i, j+1}$ of indexes $\left(q_{0}, r_{0}\right)=$ $\left(\eta N_{p}+j, \eta N_{p}+j+1\right)$ are spaced with lag $D_{i, j}$, whereas for all the other pulse-pairs, we may impose the condition

$$
\sum_{l=q}^{r-1} D_{i-\eta, l} \neq D_{i, j}
$$

$(q, r) \in \mathcal{A} \backslash\left(q_{0}, r_{0}\right)$, so that the expected value of their cross correlation is zero, and then $E\left\{y_{j}[i]\right\} \approx b_{i, j} E_{w}\left(1-e^{-\gamma T_{I}}\right) d_{i}$, resulting in

$$
E\{z[i]\} \approx E_{s}\left(1-e^{-\gamma T_{I}}\right) d_{i} .
$$

Condition (19) can be accomplished only if the periodicity of the DH code is $M \geq \eta+2$, and $D_{i-\eta, q} \neq D_{i-\eta, r}$, for $q \neq r$. This means that the AcR must be provided with a minimum of $(\eta+2) N_{p}$ delay lines plus correlators, making the hardware expensive and complex. In Section III-C, we show that result (20) can also be obtained with $N_{p^{-}}$receiver branches and with a delay-hopping code of periodicity $M=1$, via an appropriate design of the chip and DH codes. 
First, we consider a DH code of $N_{p}$ elements. Then, (19) can be rewritten as

$$
\sum_{l=q}^{r-1} D_{l} \neq D_{j}
$$

where $D_{l}=D_{l \bmod N_{p}}$. Because the DH code is repeated every $N_{p}$ frames, it follows that the adjacent pulse-pairs of indexes $(\tilde{q}, \tilde{r}) \in \mathcal{Q}=\left\{(q, q+1) \mid q=n N_{f}+j, n=0 \ldots \eta+1\right\}$ are spaced with lag $D_{j}$, and then, from (15) and (16), follows:

$$
E\left\{\left[\mathbf{Y}_{j}\right]_{\tilde{q}+1, \tilde{r}+1}\right\} \approx E_{w} e^{(n-\eta) \gamma T_{s}}\left(1-e^{-\gamma T_{I}}\right)
$$

for $n=0, \ldots, \eta$, and equal to 0 for $n=\eta+1$ (since $T_{I}<T_{s}$, pulses $a_{i+1, j}$ and $a_{i+1, j+1}$ do not fall in the observation window). Since the multiplication of the pulse amplitudes associated with $\left[\mathbf{Y}_{j}\right]_{\tilde{q}+1, \tilde{r}+1}$, i.e., $a_{i-\eta+n, j} a_{i-\eta+n, j+1}=b_{i-\eta+n, j} d_{i-\eta+n}$, is linearly data dependent, the linear ISI is a nonzero mean random variable.

For all the elements $(q, r) \in \mathcal{A} \backslash \mathcal{Q}$, the condition (21) can be satisfied, and since the product $a_{i-\eta, q} a_{i-\eta, r}$ is either data independent or nonlinearly data dependent, a DH code with periodicity $M=1$ and with properties (21) is able to suppress the expected values of all the data independent and nonlinearly data dependent elements of the quadratic form $\mathbf{a}^{T}[i] \mathbf{Y}_{j} \mathbf{a}[i]$, i.e., $E\left\{\xi_{j}\right\}=0, E\left\{\mathbf{H}_{j}\right\}=\mathbf{0}$. Notice that (21) extends the obvious condition [4]

$$
D_{q} \neq D_{j}
$$

with a significant improvement of the BER, as shown in Fig. 2. Further comments are postponed to Section III-C.

\section{B. Chip Code Design}

Now, we show that a chip code of periodicity $L N_{p}$, in combination with the DH code (21), is capable to suppress the expected value of the linear ISI terms, till $(L-1)$ symbols in the past, with $L \leq N_{p}$. Let us denote with $\mathbf{b}[l] \triangleq\left[b_{l, 0} b_{l, 1} \ldots b_{l, N_{p}-1}\right]^{T}$, the chip code vector associated to the symbol $l$. From (11) and (22), we obtain

$$
E\left\{h_{i, j}(-l)\right\} \approx b_{i-l, j} E_{w} e^{-l \gamma T_{s}}\left(1-e^{-\gamma T_{I}}\right)
$$

where $l=0, \ldots, \eta$. Since $\mathbf{h}_{i}=\sum_{j=0}^{N_{p}-1} b_{i, j} \mathbf{h}_{i, j}$, we have

$$
E\left\{h_{i}(-l)\right\} \approx E_{w}\left(1-e^{-\gamma T_{I}}\right) e^{-l \gamma T_{s}} \mathbf{b}[i]^{T} \cdot \mathbf{b}[i-l] .
$$

The above result leads to choose a chip code with the following properties:

$$
\mathbf{b}[l] \cdot \mathbf{b}\left[l^{\prime}\right]=0 \text { if } l \neq l^{\prime}
$$

and (25) becomes

$$
\begin{array}{r}
E\left\{h_{i}(-l)\right\} \\
\approx \begin{cases}0, & \text { if }(i-l) \bmod L \neq 0 \\
E_{s}\left(1-e^{-\gamma T_{I}}\right) e^{-l \gamma T_{s},} & \text { if }(i-l) \bmod L=0 .\end{cases}
\end{array}
$$

Each vector $\mathrm{b}[l]$ consists of $N_{p}$ elements, with polarity \pm 1 . Thus, the maximum number of orthogonal vectors is $L_{\max }=$ $N_{p}$. We conclude that the FD-AcR operating with $N_{p}$ pulses per

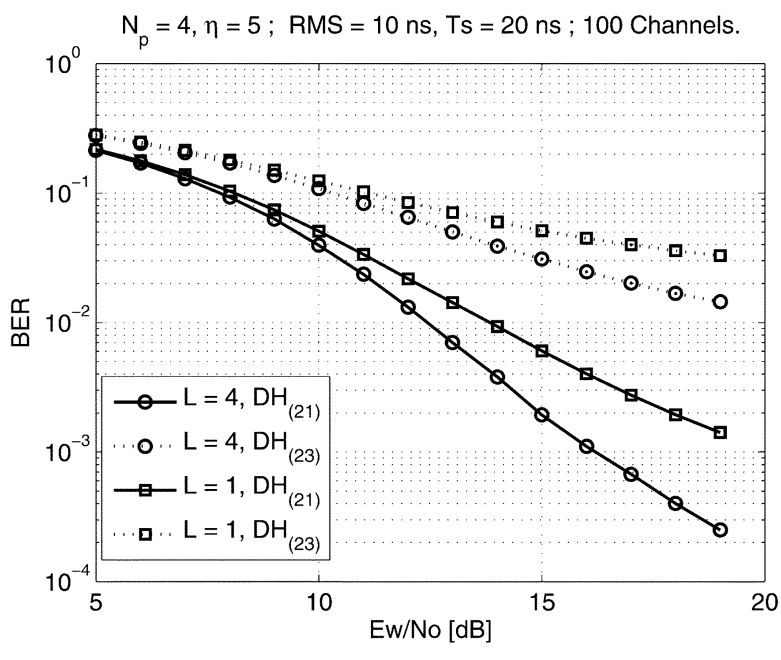

Fig. 2. Performance improvement via DH and chip codes design, averaged over 100 independent channel realizations. Comparison between a random DH code with properties (23) and DH code with properties (21), and between chip codes of periodicity $L N_{p}$.

symbol can suppress the expected values of $N_{p}-1$ interfering symbols. If $N_{p} \geq \eta+1$, we obtain $E\{\mathbf{h}\} \approx E_{s}\left(1-e^{-\gamma T_{I}}\right)$. $\left[\begin{array}{lllll}0 & \ldots & 0 & 1 & 0\end{array}\right]$, and therefore

$$
E\{z[i]\} \approx E_{s}\left(1-e^{-\gamma T_{I}}\right) \cdot d_{i} .
$$

\section{Bit-Error Rate (BER) Results}

We considered a system operating at $50 \mathrm{Mbit} / \mathrm{s}$, in a typical indoor environment characterized by a RMS delay spread of $10 \mathrm{~ns}$, resulting in $\eta \approx 5$ interfering symbols. Simulated BER results, averaged over 100 independent channel realizations, are plotted in Fig. 2 and visualize and quantify the performance improvement. The four curves plotted in the figure shows the impact of the DH and chip codes design on interference suppression. The dotted line with the square marks represents the performance of a conventional FD-AcR, with chip code periodicity equal to $N_{p}$, and with a random DH code satisfying only the conditions $D_{q} \neq D_{j}$. The improvement obtained by the reduction of the linear ISI is shown by the dotted line with empty circle marks, which refers to a chip code with periodicity $L N_{p}$, $L=4$. In a "zero-threshold" detector, which does not estimate the bias-term, an outstanding improvement is obtained by a DH code with properties (21), $L=1$, as pointed out by the solid line with square marks. Such a code is extremely effective in nonlinear ISI and bias-interference reduction, which are not mitigated by the DH code (23). Once the interference of these terms is reduced, a further noticeable improvement in the BER curve is achieved by the linear ISI reduction, via the chip code (26), as illustrated by the solid line with circle marks.

\section{Number of Pulses Per Symbol}

AcRs, when compared with optimum receivers, suffer from enhanced noise power due to a noise-to-noise term, arising from the noisy template adopted for demodulation. In order to reduce this term, a modified AcR has been proposed in [5], where the 
noise in the signal template is reduced by averaging over previous reference pulses. A drawback of this scheme is that for each extra pulse used in the averaged reference, an additional delay line is required. Furthermore, the delay between data and reference pulse becomes increasingly longer, making the implementation of an accurate analog delay line very difficult, if not impossible. This solution, theoretically efficient, is therefore discarded in the following considerations for practical reasons and the AcR as presented in Section II-B is considered. For this receiver, the noise-to-noise term is shown to be linearly proportional to the number of transmitted pulses per symbol [6], [13], [14], and therefore the transmission of one pulse per symbol is the optimum choice to reduce the noise power.

On the other hand, in Section III, we showed that with $N_{p}$ pulses per symbol it is possible to form a chip code of length $L=N_{p}$ symbols such that the expectation of $N_{p}-1$ linear ISI terms is reduced to zero, with a considerable gain in terms of BER. Thus, for $\eta$ interfering symbols, the transmission of $N_{p}=\eta+1$ pulses per symbol is the favorable choice to reduce the ISI effects.

In this section, we want to derive the number of pulses per transmitted symbol, which minimizes the BER, under the constraint of a fixed energy per symbol $E_{s}=N_{p} E_{w}$ and a fixed symbol duration $T_{s}$. We define $\mathbf{d}^{+}[i] \triangleq\left[d_{i-\eta} \ldots d_{i-1} 1 d_{i+1}\right]^{T}$, as the data sequence where $d_{i}=+1$, and

$$
\hat{z}^{+}[i]=\xi_{i}+\mathbf{h}_{i}^{T} \mathbf{d}^{+}[i]+\left(\mathbf{d}^{+}\right)^{T}[i] \mathbf{H}_{i} \mathbf{d}^{+}[i]+v[i]
$$

the AcR sampled output, including the noise term $v[i]$, given that $d_{i}=+1$ was transmitted. We can assume that the noise term $v[i]$ is Gaussian distributed [5], [13], and that the bias term $\xi_{i}$ is known at the receiver. Then, we can write the probability of error, conditioned to the data sequence $\mathbf{d}^{+}[i]$, and conditioned to the channel, embedded in the parameters $\mathbf{h}_{i}$ and $\mathbf{H}_{i}$, as

$$
P\left(e \mid \mathbf{h}_{i}, \mathbf{H}_{i}, \mathbf{d}^{+}[i]\right)=Q\left(\frac{z^{+}[i]}{\sigma_{v}}\right)
$$

where $\sigma_{v}^{2}$ is the noise variance, conditioned to the channel, and $z^{+}[i]=\hat{z}^{+}[i]-\xi_{i}-v[i]$ is the noise-free, bias-free AcR sampled output, given that $d_{i}=+1$ was transmitted. The variance of the noise at the output of the AcR under ISI has been evaluated in [15], and can be written as ${ }^{1}$

$$
\sigma_{v}^{2} \approx \frac{N_{0}}{2} E_{s} \kappa+N_{p}\left(\frac{N_{0}}{2}\right)^{2} T_{I} N_{2}
$$

where $N_{0} / 2$ is the noise power spectral density, $\kappa$ is a channel gain related parameter, ${ }^{2}$ and $N_{2} \triangleq \int_{-\infty}^{\infty}|F(f)|^{4} d f, F(f)$ being the frequency response of the filter $f(t)$ at the receiver input. In case of an ideal rectangular filter $N_{2}=W$.

The dependence of the instantaneous signal-to-noise-ratio $z^{+}[i] / \sigma_{v}$ on $N_{p}$ follows from (30) and from the discussion presented in Section III. By considering the terms of $\mathbf{H}_{i}$ independent from $N_{p}$, and by recalling that changing the number of pulses per symbol impacts essentially on the expected value of the linear ISI coefficients, for the purpose of our derivation, we

${ }^{1}$ Strictly speaking the noise power is weakly data dependent, as shown in (22), [15]. However, a simplified noise model can assume a data-independent noise variance.

${ }^{2}$ The $\kappa$ parameter equals 1 in the AWGN channel, represents the energy in the ISI-free multipath channel (see, for instance, [13, eq. (11)]), and it is implicitly defined in [6] and [15] for ISI channels. can use the approximation $z^{+}[i] \approx E\left\{\mathbf{h}_{i}\right\}^{T} \mathbf{d}^{+}[i]$. Then, we define $\rho\left(N_{p}\right) \triangleq E\left\{\mathbf{h}_{i}\right\}^{T} \mathbf{d}^{+}[i] / \sigma_{v}\left(N_{p}\right)$, where $\sigma_{v}^{2}(n)$ denotes the noise power for $N_{p}=n$, and by applying (25), we obtain

$$
\rho\left(N_{p}\right)=\frac{1+\sum_{l=N_{p}}^{\eta} d_{i-l} e^{-l \gamma T_{s}}}{\sigma_{v}\left(N_{p}\right)} E_{s}\left(1-e^{-\gamma T_{I}}\right)
$$

where a chip code with periodicity $L=N_{p}$ symbols and properties (26) is employed. The number of pulses per symbol $\hat{N}_{p}$ which minimizes the probability of error can be therefore found as

$$
\hat{N}_{p}=\arg \max _{N_{p}}\left\{\rho\left(N_{p}\right)\right\} .
$$

Inspired by simulated results, which pointed out $\hat{N}_{p}=1$ for many configurations of system parameters, we want to find under which conditions holds the following inequality:

$$
\rho(1)-\rho\left(N_{p}\right)>0
$$

which, by using (31), can be rewritten as

$$
\Delta \sigma_{v}\left[1+\sum_{l=N_{p}}^{\eta} d_{i-l} e^{-l \gamma T_{s}}\right]+\sigma_{v}\left(N_{p}\right) \sum_{l=1}^{N_{p}-1} d_{i-l} e^{-l \gamma T_{s}}>0
$$

where $\Delta \sigma_{v} \triangleq \sigma_{v}\left(N_{p}\right)-\sigma_{v}(1)$. The left side of the inequality above depends on the data sequence, and it has a minimum value for the data sequence $\mathbf{d}^{+}[i]=[-1 \ldots-1+1-1]$, given by $\Delta \sigma_{v}\left(1-e^{-\gamma T_{s}}-e^{-N_{p} \gamma T_{s}}\right)-\sigma_{v}\left(N_{p}\right)\left(e^{-\gamma T_{s}}-e^{-N_{p} \gamma T_{s}}\right)>0$ which can be rewritten as

$$
\sigma_{v}\left(N_{p}\right)\left(1-2 e^{-\gamma T_{s}}\right)>\sigma_{v}(1)\left(1-e^{-\gamma T_{s}}-e^{-N_{p} \gamma T_{s}}\right) .
$$

Defining $\chi=\sigma_{v}(1) / \sigma_{v}\left(N_{p}\right)$, where $1 / N_{p}<\chi<1$ by definition, and upper bounding the right side of the inequality above with $\sigma_{v}(1)\left(1-e^{-\gamma T_{s}}\right)$, the sufficient condition to ensure that the transmission of one pulse per symbol minimizes the probability of error is

$$
\gamma T_{s}>\ln \left(\frac{2-\chi}{1-\chi}\right) .
$$

The right side of inequality (35) is monotonically increasing with $\chi$, and diverges to infinity for $\chi \rightarrow 1$. Indeed, $\chi=1$ is the limit obtained for $E_{s} \rightarrow \infty$, when the noise-to-noise term becomes negligible and the total noise power is independent from the number of pulses $N_{p}$. Obviously, in this situation, the tradeoff between ISI suppression and noise enhancement has no sense anymore, as increasing the number of pulses will only reduce the ISI. However, since the value $\chi=1$ is not reached in any practical case (the noise-to-noise term cannot be totally suppressed or neglected by transmitting infinite energy), the right side of (35) is limited. By assigning realistic values to the parameters appearing in (30), it is possible to get an impression on the range of $\chi$ values. Assuming for instance a signal bandwidth $W=2 \mathrm{GHz}$, an integration time $T_{I}=$ $T_{s}=24 \mathrm{~ns}$, and $E_{s} / N_{0}=20 \mathrm{~dB}, \kappa=1$, it turns out that the power of the signal-to-noise term [i.e., the first term in (30)] is four times larger than the noise-to-noise power [i.e., the second term in (30)], resulting in $\chi \leq 5 / 6$. Substituting this value in (35), it follows that for $\gamma T_{s}>1.95$ the transmission of one pulse per symbol is the optimum choice in the minimum BER 


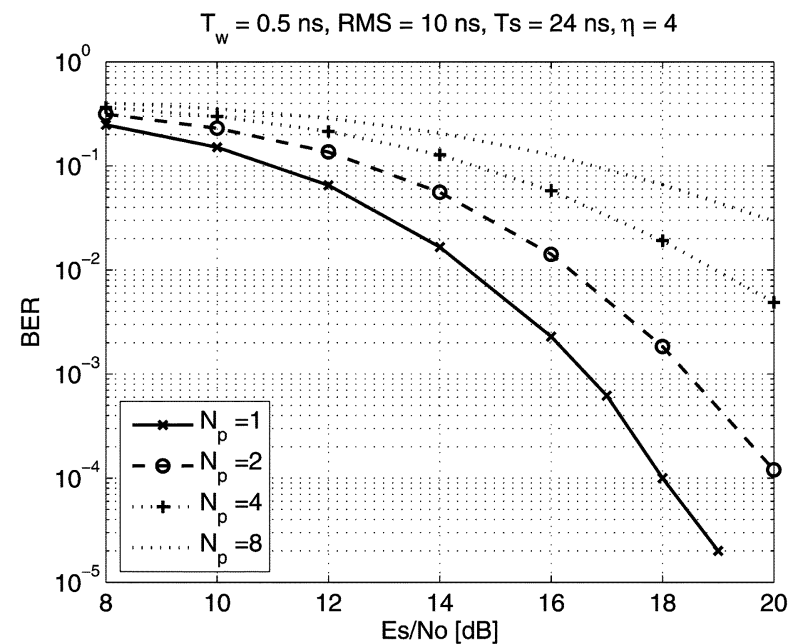

Fig. 3. Performance as function of the number of pulses per symbol $N_{p}$.

sense. However, the reverse is not true, i.e., $N_{p}=1$ can be the optimum choice even if (35) is not satisfied. Fig. 3 plots the simulated BER for different number of transmitted pulses per symbol, in a multipath channel with RMS delay spread of $10 \mathrm{~ns}$, and symbol duration of $24 \mathrm{~ns}$, corresponding to a symbol rate $R_{s} \approx 41 \mathrm{Mbit} / \mathrm{s}$, and to $\gamma T_{s}=2.4$. The integration time is chosen to be equal to the symbol duration, and signal bandwidth approximately equal to $2 \mathrm{GHz}$. The symbol energy $E_{s}$ does not depend on the transmitted number of pulses, and the pulse energy is $E_{w}=E_{s} / N_{p}$. The transmission of one pulse per symbol is the optimum choice, as indicated by (35), and the performance gain is substantial, even in a scenario where the ISI is pronounced ( $\eta=4$ interfering symbols). We observe that doubling $N_{p}$ induces a $2 \mathrm{~dB}$ loss, and a more pronounced difference in terms of BER performance is expected by increasing $\gamma T_{s}$. The same figure also confirms the validity of the bound on $\gamma T_{s}$.

Notice that this gain is obtained in parallel to a reduced complexity of the receiver hardware, since for $M=1$ and $N_{p}=1$ only one delay-line and one correlator need to be implemented. Unfortunately, the periodicity of the transmitted signal creates undesired peaks in the signal spectrum. Furthermore, the multiple-access capabilities can be compromised, as increasing the number of pulses per symbol can reduce the effect of collisions with pulses of other users.

Nevertheless, the outstanding gain showed in Fig. 3 motivates further discussion. First of all, in a multipath channel, the event that two pulses from different users arrive at the receiver at the same time is not so catastrophic as in a single-path channel, since the channel itself decorrelates the two signals [16]. Next, as the single-user case is investigated in this paper, multipleaccess is not an urgent issue here. Then, we show in Section V how to further improve the system performance by applying the DH code at the symbol level, which also results in a smoothing of the signal spectrum.

\section{ONE-PULSE-PER-Symbol ACR}

With only one transmitted pulse per symbol, the frame-level disappears, and we can refer to this scheme as symbol differen-

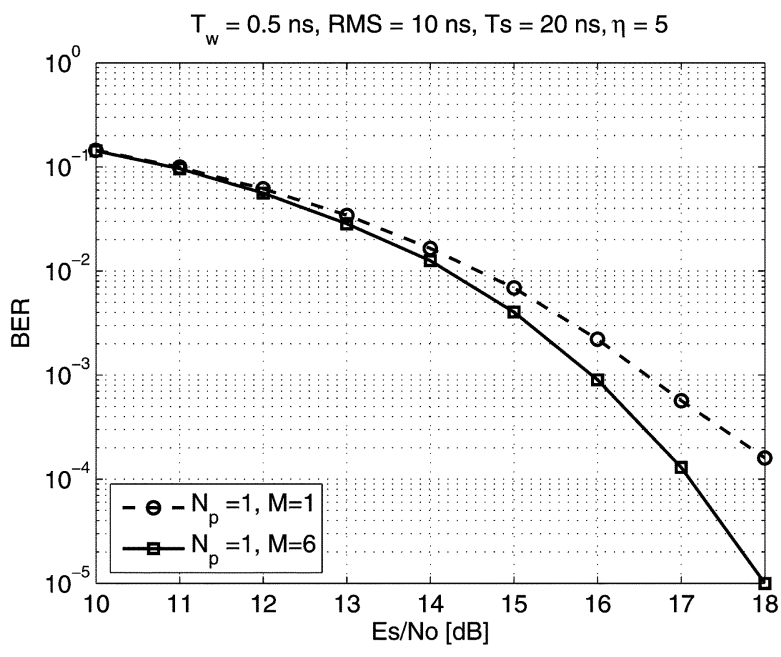

Fig. 4. Performance comparison between DH codes of length $M=6$ and $M=1$ symbols.

tial modulation. The first part of the signal model presented in Section II can be directly rewritten as

$$
\tilde{r}(t)=\sum_{n=-\infty}^{\infty} a_{n} g\left(t-t_{n}\right)+v(t)
$$

where $a_{n+1}=a_{n} d_{n}$ and $t_{n}=n T_{s}+c_{n} T_{c}$. The chip code has been suppressed because it is irrelevant, and the TH code is repeated every $M$ symbols, with the notation $c_{n}=c_{n \bmod M}$. The differential autocorrelation receiver (D-AcR) consists of $M$ correlators, and the observed variable without noise is given as $z[i]=\mathbf{a}^{T}[i] \mathbf{Z}_{i} \mathbf{a}[i]$, where

$$
\left[\mathbf{Z}_{i}\right]_{q+1, r+1}=\int_{t_{i}}^{t_{i}+T_{I}} g\left(t-t_{i-\eta+q}\right) g\left(t-t_{i-\eta+r}+D_{i}\right) d t
$$

and $q, r=0, \ldots, \eta+1$. The pulse polarity vector is given as

$$
\mathbf{a}[i]=\left[a_{i-\eta} \ldots a_{i+1}\right]^{T}
$$

and the amplitude of each pulse is given by

$$
a_{i-\eta+q}=a_{i-\eta} \prod_{l=0}^{q-1} d_{i-\eta+l} .
$$

By applying the results derived in Section III-A, we can find a DH code of length $M=\eta+1$, such that $E\{z[i]\}=d_{i} E_{s}(1-$ $\left.e^{-\gamma T_{I}}\right)$. The performance of a D-AcR with DH code of periodicity $M=\eta+1$ and $M=1$ are compared in Fig. 4. The gain obtained with a longer $\mathrm{DH}$ code becomes more significant for high values of the SNR $E_{s} / N_{0}$, where the ISI is the most important deteriorating effect.

\section{CONCLUSION}

In this paper, we show how to reduce the effects of nonlinear ISI and the bias term, nuisance parameters of autocorrelation receivers, by adopting a DH code which uncorrelates the pulses of the received signal with the pulses of the delayed (template) signal, except for the pulse-pair carrying the differentially modulated bit. This is accomplished by a set of DH codes which satisfy a number of conditions pointed out in the paper. Further- 
more, for a UWB system transmitting $N_{p}$ pulses per symbol, we demonstrate the capability of a chip code with periodicity $L \leq N_{p}$ symbols to suppress the expected values of $L-1$ linear ISI coefficients.

Since in conventional AcRs the noise power is linearly proportional to the transmitted number of pulses, large values of $N_{p}$ lead both to ISI mitigation, and to an increased level of the noise power. We prove that for bit rates lower than an upper bound depending on the RMS delay spread of the channel and on the noise power, the transmission of one pulse per symbol minimizes the probability of error, whereas the reverse is not true, i.e., the transmission of one pulse per symbol could be the optimum choice also for higher bit rates. Despite the fact that a large number of pulses per symbol might be required in a multiple-access system, the outstanding gain shown by the onepulse-per-symbol differential AcR motivates its applicability at least in a single-user scenario.

\section{REFERENCES}

[1] M. Pausini, G. J. M. Janssen, and K. Witrisal, "Delay hopping and chip codes design for a frame differential UWB autocorrelation receiver," in Proc. IEEE Int. Conf. Commun., Seoul, Korea, May 2005, pp. 417-422.

[2] C. Rushforth, "Transmitted-reference techniques for random or unknown channels," IEEE Trans. Inf. Theory, vol. 10, pp. 39-42, Jan. 1964.

[3] R. Gagliardi, "A geometrical study of transmitted reference communication system," IEEE Commun. Lett., pp. 118-123, Dec. 1964.

[4] R. Hoctor and H. Tomlinson, "Delay-hopped transmitted-reference RF communications," in Proc. IEEE Conf. Ultra Wideband Syst. Technol., Baltimore, MD, May 2002, pp. 265-270.

[5] J. Choi and W. Stark, "Performance of ultra-wideband communications with suboptimal receivers in multipath channels," IEEE J. Sel. Areas Commun., vol. 20, pp. 1754-1766, Dec. 2002.

[6] K. Witrisal, C. Krall, G. Leus, and M. Pausini, "Equivalent system model and equalization of differential impulse radio UWB systems," IEEE $J$. Sel. Areas Commun., pp. 1851-1862, Sep. 2005.

[7] M. Ho, V. S. Somayazulu, J. Foerster, and S. Roy, "A differential detector for an ultra-wideband communications system," in Proc. IEEE Veh. Technol. Conf.-Spring, 2002, pp. 1896-1900.

[8] Y.-L. Chao and R. A. Scholtz, "Optimal and suboptimal receivers for ultra-wideband transmitted reference systems," in Proc. IEEE Global Telecommun. Conf., San Francisco, CA, Dec. 2003, pp. 759-763.

[9] M. Pausini, G. J. M. Janssen, and K. Witrisal, "Analysis of ISI for an IR-UWB symbol-differential autocorrelation receiver," in Proc. IEEE Veh. Technol. Conf., Los Angeles, CA, Sep. 2004, pp. 1213-1217.

[10] V. J. Mathews and G. L. Sicuranza, Polynomial Signal Processing. Chichester, U.K.: Wiley, 2000.

[11] K. Witrisal, M. Pausini, and A. Trindade, "Multiuser interference and inter-frame interference in UWB transmitted reference systems," in Proc. Int. Workshop UWB Syst. Joint with Conf. UWB Syst. Technol., Joint UWBST \& IWUWBS, Kyoto, Japan, May 2004, pp. 96-100.

[12] K. Witrisal and M. Pausini, "Impact of multipath propagation on impulse radio UWB autocorrelation receivers," in Proc. IEEE Int. Conf. UltraWideband, Zurich, Switzerland, Sep. 2005, pp. 485-490.

[13] T. Q. S. Quek and M. Z. Win, "Analysis of UWB transmitted-reference communication systems in dense multipath channels," IEEE J. Select. Areas Commun., pp. 1863-2174, Sep. 2005.

[14] N. He and C. Tepedelenlioglu, "Performance analysis of noncoherent UWB receivers at different synchronization levels," in Proc. IEEE Global Telecommun. Conf., Dallas, TX, Dec. 2004, pp. 3517-3521.
[15] K. Witrisal and M. Pausini, "Equivalent system model of ISI in a frame-differential IR-UWB receiver," in Proc. IEEE Global Telecommun. Conf., Dallas, TX, Dec. 2004, pp. 3505-3510.

[16] Y. Chao and R. Scholtz, "Multiple access performance of ultra-wideband transmitted reference systems in multipath environments," in Proc. IEEE Wireless Commun. Netw. Conf., Atlanta, GA, Mar. 2004, pp. 1788-1793.

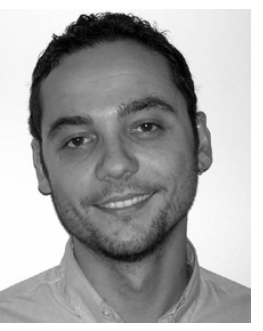

Marco Pausini (S'03) received the Dr. Ing. degree in telecommunication engineering from the University of Bologna, Bologna, Italy, in 2001. He is currently working towards the Ph.D. degree at the Department of Electrical Engineering, Technical University of Delft, Delft, The Netherlands.

From June 2001 to June 2002, he was with the Center of Excellence For Research, Innovation, Education and Industrial Laboratories Partnership, Politecnico di Milano, Italy (CEFRIEL-ICT), working on smart antenna design for WCDMA systems. His research interests include communication theory, channel modeling, and ultrawide-bandwidth (UWB) communications systems.

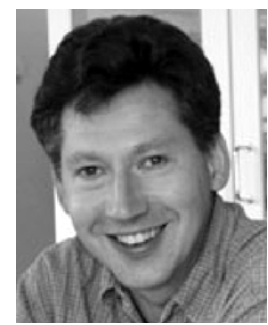

Gerard J. M. Janssen (M'93) received the M.Sc.E.E. degree from Eindhoven University of Technology, Eindhoven, The Netherlands, in 1986, and the Ph.D. degree from the Delft University of Technology, Delft, The Netherlands, in 1998.

In 1986, he joined the Physics and Electronics Laboratory of the Dutch Organization of Applied Scientific Research (TNO), where he was involved in radar-cross-section modeling, radio direction finding, interference cancellation, and wideband propagation measurements. $\mathrm{He}$ is currently an Associate Professor in the Wireless and Mobile Communications Group, Delf University of Technology. His research interests are in wireless communication, especially narrowband multiuser detection, digital modulation techniques, channel modeling, diversity techniques and ultra-wideband communications.

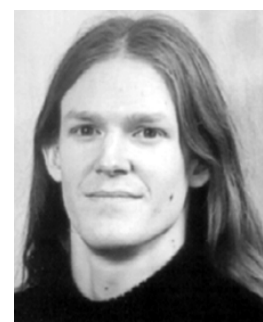

Klaus Witrisal (S'98-M'03) was born in Graz, Austria, in 1972. He received the Dipl.-Ing. degree in electrical engineering from Graz University of Technology, Graz, in 1997 and the Ph.D. degree (cum laude) from the Delft University of Technology, Delft, The Netherlands, in 2002

He was appointed as a Research Engineer at the Delft University of Technology from 1997 to 2001, working on frequency-domain channel characterization and OFDM transmission technology. From January to October 2002, he was a Project Leader with AVL, Graz, Austria. Currently, he is an Assistant Professor at the Signal Processing and Speech Communication Laboratory (SPSC), Graz University of Technology, where he runs a number of research projects in the area of ultra-wideband (UWB) communications, in cooperation with national and international partners. Among these activities is the project EUROPCOM funded by the European Commission, which aims to develop a communications and positioning system for emergency services. His research interests are in signal processing for broadband and UWB wireless communications, propagation channel modeling, and positioning. 